\title{
Efficacy and Safety of Methylnaltrexone for the Treatment of Opioid-Induced Constipation: A Meta-analysis of Randomized Controlled Trials
}

\author{
Ying-Ying Zhang $\cdot$ Rong Zhou · Wan-Jie Gu (D)
}

Received: December 6, 2020 / Accepted: January 20, 2021 / Published online: February 11, 2021

(c) The Author(s) 2021

\begin{abstract}
Introduction: Opioid-induced constipation (OIC) is a distressing side effect during opioid analgesia and is mainly mediated by gastrointestinal $\mu$-opioid receptors. Methylnaltrexone, a peripheral $\mu$-opioid receptor antagonist with restricted ability to cross the blood-brain barrier, may alleviate OIC without reversing analgesia. We performed a meta-analysis to assess the efficacy and safety of methylnaltrexone for the treatment of OIC.

Methods: This meta-analysis was registered in PROSPERO (CRD42020187290). We searched PubMed, Embase, and Cochrane Library for randomized controlled trials that compared methylnaltrexone with placebo for the treatment of OIC. Relative risks (RR) and 95\% confidence interval (CI) were pooled using a random-effects model. We used the GRADE approach to assess the certainty of the evidence. Results: Eight trials with 2034 participants were included. Compared with placebo, methylnaltrexone significantly increased rescue-free bowel movement (RFBM) within $4 \mathrm{~h}$ after the first dose (eight trials; 1833 participants; RR 3.74, 95\% CI 3.02-4.62; high-
\end{abstract}

Y.-Y. Zhang · R. Zhou · W.-J. Gu ( $\bowtie)$

Department of Anesthesiology, Nanjing Drum

Tower Hospital, The Affiliated Hospital of Nanjing

University Medical School, Nanjing 210008, China

e-mail:wanjiegu@hotmail.com certainty evidence), RFBM within $24 \mathrm{~h}$ after the first dose (two trials; 614 participants; RR 1.98, 95\% CI 1.52-2.58; moderate-certainty evidence), and $\mathrm{RFBM} \geq 3$ times per week (three trials; 1,396 participants; RR 1.33, 95\% CI 1.17-1.52; moderate-certainty evidence) and decreased need to take rescue laxatives (three trials; 807 participants; RR 0.73, 95\% CI 0.63-0.85; moderate-certainty evidence). For safety outcomes, there was no difference in any adverse events between the two groups (eight trials; 2034 participants; RR 1.11, 95\% CI 0.99-1.23; moderate-certainty evidence), including diarrhea, nausea, vomiting, and flatulence; but for the most commonly reported adverse events, the abdominal pain was higher in methylnaltrexone group than that in placebo group (six trials; 1813 participants; RR 2.30, 95\% CI 1.29-4.08; moderate-certainty evidence).

Conclusion: Methylnaltrexone is an effective and safe drug for the treatment of OIC, but the safety of abdominal pain should be considered.

Keywords: Meta-analysis; Methylnaltrexone; Opioid-induced constipation 


\section{Key Summary Points}

Opioid-induced constipation (OIC) is a distressing side effect during opioid analgesia.

Methylnaltrexone is an effective and safe drug for the treatment of OIC.

The safety of abdominal pain should be considered.

\section{DIGITAL FEATURES}

This article is published with digital features, including a summary slide, to facilitate understanding of the article. To view digital features for this article go to https://doi.org/10.6084/ m9.figshare.13614500.

\section{INTRODUCTION}

Opioid-induced constipation (OIC) is the most common side effect during opioid analgesia in patients with advanced illness including incurable cancer or other terminal diseases. According to Rome IV, OIC is defined as new or deteriorating constipation when initiating, changing, or increasing opioid therapy, which must include two or more of the following: straining, lumpy or hard stools, sensation of incomplete evacuation, sensation of anorectal blockage, use of manual maneuvers to facilitate defecation, and fewer than three spontaneous bowel movement per week [1]. Results showed that $>85 \%$ of cancer and $>40 \%$ of non-cancer patients treated with opioids experience symptoms of OIC [2]. Different from other complications of opioids such as nausea or vomiting, tolerance to constipation develops very slowly. In addition to increasing hospitalization and healthcare costs [3], OIC may cause patients to become intolerant to opioids, thus greatly compromising the analgesic effect of opioids and leading to a serious decline in quality of life

[4]. The first-line strategy to treat OIC is a prophylactic regimen that involves increased fluid and fiber intake, exercise, stool softeners, and laxatives. However, at present, there is a lack of high-quality evidence to confirm the effectiveness of these treatment regimens $[5,6]$. The second-line treatment, which includes peripherally acting $\mu$-opioid receptor antagonists, can be considered when patients have recalcitrant symptoms.

Methylnaltrexone, a pure peripheral $\mu$-opioid receptor antagonist, is a quaternary compound created by adding a methyl group to the opioid antagonist naltrexone [7]. Since the methyl group restricts its ability to cross the blood-brain barrier, methylnaltrexone can alleviate OIC effectively without weakening centrally mediated analgesia. So far, trials reporting the effect of methylnaltrexone on the treatment of OIC have conveyed conflicting results. Furthermore, due to modest sample size, these individual trials were not adequately powered to detect the true effect.

The aim of this study was to investigate the efficacy and safety of methylnaltrexone for the treatment of OIC by performing a metaanalysis.

\section{METHODS}

\section{Compliance with Ethics Guidelines}

This article is based on previously conducted studies and does not contain any studies with human participants or animals performed by any of the authors.

\section{Protocol and Registration}

The meta-analysis was performed in accordance with the Cochrane Handbook for Systematic Reviews of Interventions [8] and is reported in compliance with the PRISMA (Preferred Reporting Items for Systematic Reviews and Meta-Analyses) statement [9]. This meta-analysis was prospectively registered in PROSPERO (CRD42020187290). 


\section{Data Sources and Search Strategy}

We searched PubMed, Embase, and Cochrane Library from inception to May 19, 2020, without any restrictions. Search terms included: methylnaltrexone, opioid*, opioid-induced constipation, intestinal dysfunction, bowel dysfunction, gut motility, and rescue-free bowel movement. The reference lists of included trials were scanned for potential eligible articles. Additionally, we reviewed conference abstracts for unpublished work.

\section{Study Selection and Eligibility Criteria}

Two authors (YYZ and WJG) independently carried out the study selection based on predefined inclusion and exclusion criteria. Disagreements were resolved by discussion. We included randomized controlled trials that compared the efficacy and safety of methylnaltrexone with placebo for the treatment of OIC in adults who received opioid therapy. We excluded trials with healthy volunteers as participants.

\section{Data Extraction and Outcomes Assessment}

We developed a data extraction sheet in standardized Excel (Microsoft Corporation, Redmond, WA, USA). Two authors (YYZ and WJG) independently extracted data from included trials. Discrepancies were handled by discussion. The following information was extracted from each trail: author, year, country, population, sample size, drug regimen (route and dosage), and outcome.

The primary efficacy outcome was rescuefree bowel movement (RFBM) within $4 \mathrm{~h}$ after first dose (RFBM was defined as a bowel movement without use of any rescue medication or procedure within $4 \mathrm{~h}$ before the bowel movement). The secondary efficacy outcomes included RFBM within $24 \mathrm{~h}$ after the first dose, $\mathrm{RFBM} \geq 3$ times per week, and need to take rescue laxatives. The primary safety outcome was any adverse events, which was defined as all treatment-related adverse events in individual trial. The secondary safety outcomes included abdominal pain, diarrhea, nausea, vomiting, and flatulence.

\section{Quality Assessment and Certainty of Evidence}

We used the Cochrane Collaboration's tool for assessing risk of bias [10]. We reviewed each trial and scored as high, low, or unclear the risk involving the following domains: random sequence generation (selection bias), allocation concealment (selection bias), blinding of participants and personnel (performance bias), blinding of outcome assessment (detection bias), incomplete outcome data (attrition bias), selective reporting (reporting bias), and other bias. Thus, trials with high risk of bias for $\geq 1$ key domains were considered to be at high risk of bias whereas trials with low risk of bias for all key domains were considered to be at low risk of bias; otherwise they were considered to be at unclear risk of bias.

We evaluated the certainty of evidence for primary and secondary outcomes according to Grading of Recommendations Assessment, Development, and Evaluation (GRADE) approach for risk of bias, inconsistency, indirectness, imprecision, and publication bias, classified as very low, low, moderate, or high [11]. Summary tables were constructed using the GRADE Profiler (version 3.6, GRADE pro).

\section{Statistical Analysis}

We calculated relative risks (RRs) with 95\% CIs for dichotomous outcomes. Meta-analyses were performed using a random-effects model accounting for clinical heterogeneity. All analyses were performed on an intention-to-treat basis. Statistical heterogeneity across trials was assessed by the Cochrane $Q$ test (with $P<0.1$ indicating significance) and quantified by the $I^{2}$ statistic $\left(I^{2}>50 \%\right.$ for a significant heterogeneity) $[12,13]$. A two-sided $P<0.05$ was considered statistically significant. All statistical analyses were performed using RevMan 5.3 (Nordic Cochrane Centre). 


\section{RESULTS}

\section{Trial Selection}

A total of 630 articles were found from electronic databases. After duplicates were removed, 419 articles had been screened for titles and abstracts. Then, 27 articles were identified for full-text review. Of these, 18 articles were excluded: seven articles were excluded because their participants were healthy volunteers; two articles were excluded because there was no relevant data; six articles were excluded because they did not use a placebo as a control group; four articles were excluded because of duplicate data. Finally, eight trials (seven full texts and one abstract) were included (Fig. 1) [14-21].

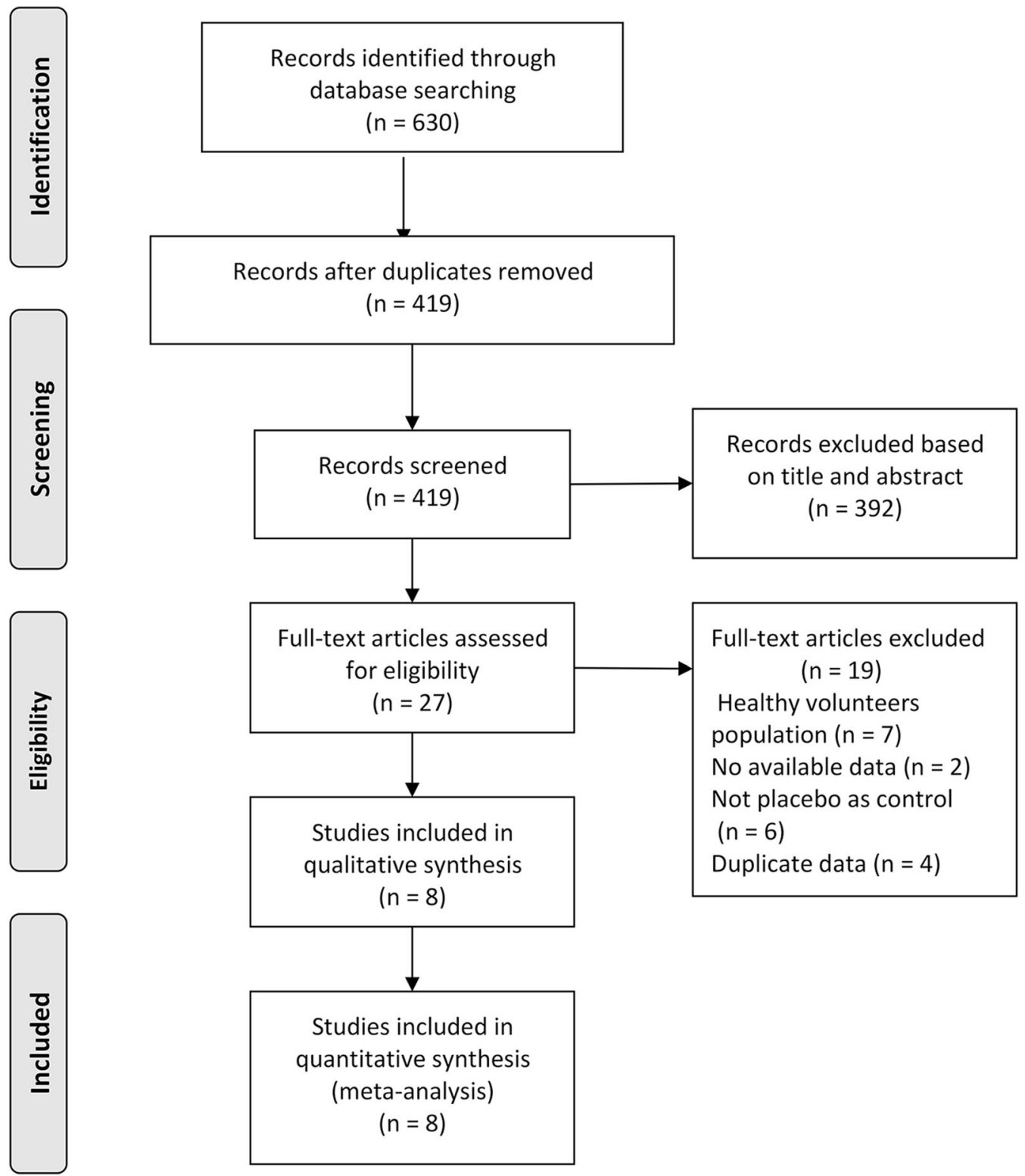

Fig. 1 PRISMA flow diagram 


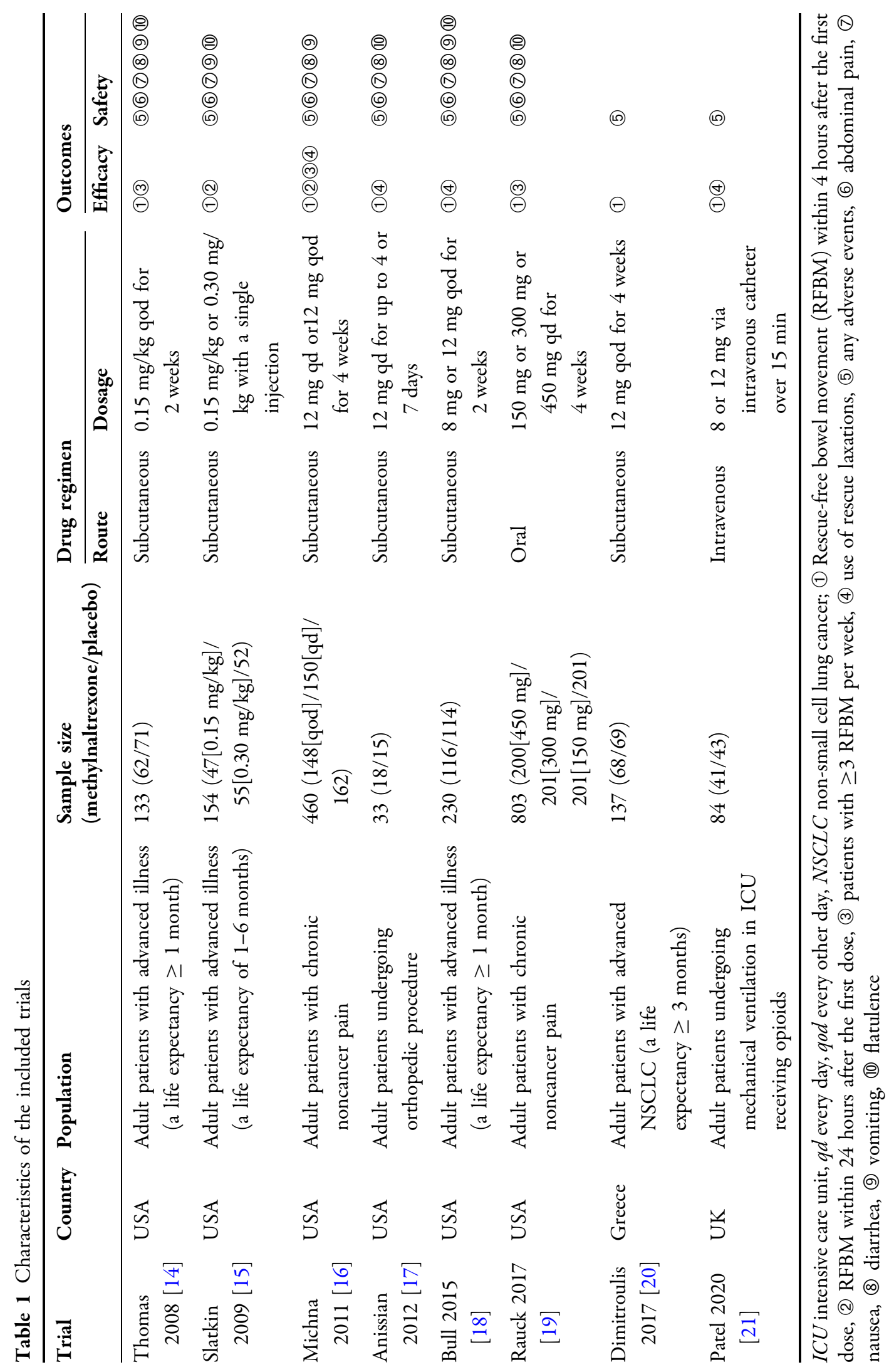




\section{Trial Characteristics}

The characteristics of the included trials are presented in Table 1 . These trials were published between 2008 and 2020. The sample size of the individual trial ranges from 33 to 803 . The population mainly involved patients with advanced illness (incurable cancer or other terminal diseases) and chronic noncancer pain. The administrated route in all trials is subcutaneous except oral in one trail and intravenous one trail. All trials reported the efficacy and safety outcomes. The details of risk-of-bias assessment for each included trial are summarized in Fig. 2. Overall, two trials were categorized as being at low risk of bias and six as being unclear risk of bias.

\section{Efficacy of Methylnaltrexone for the Treatment of OIC}

Primary Efficacy Outcome: RFBM Within $4 \mathrm{~h}$ After the First Dose

Eight trials with 1833 participants reported the primary efficacy outcome. Methylnaltrexone significantly increased RFBM within $4 \mathrm{~h}$ after the first dose compared with placebo (RR 3.74, 95\% CI 3.02-4.62; $I^{2}=0 \%$; Fig. 3).

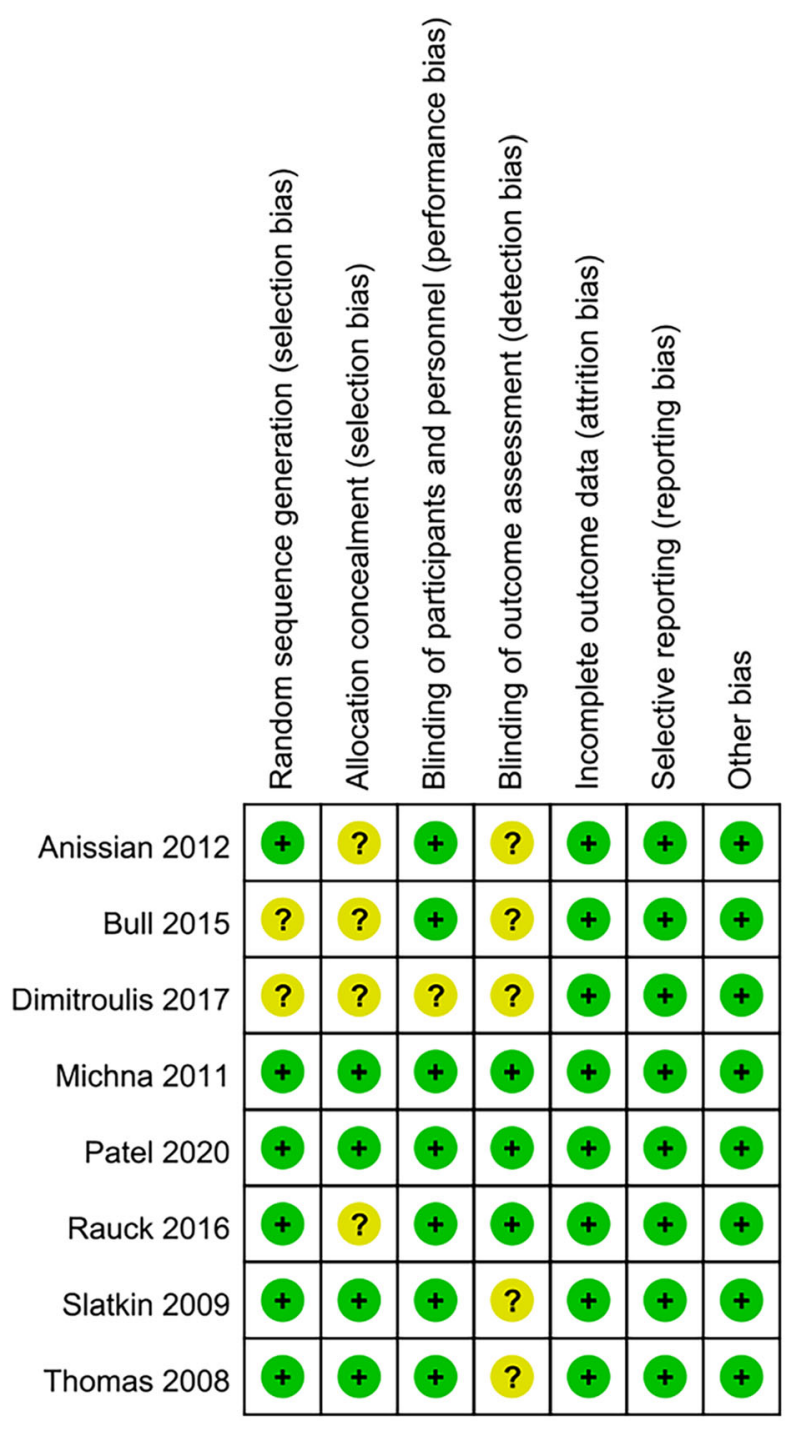

Fig. 2 Risk-of-bias summary. $+=$ low risk; ? = uncertain risk 


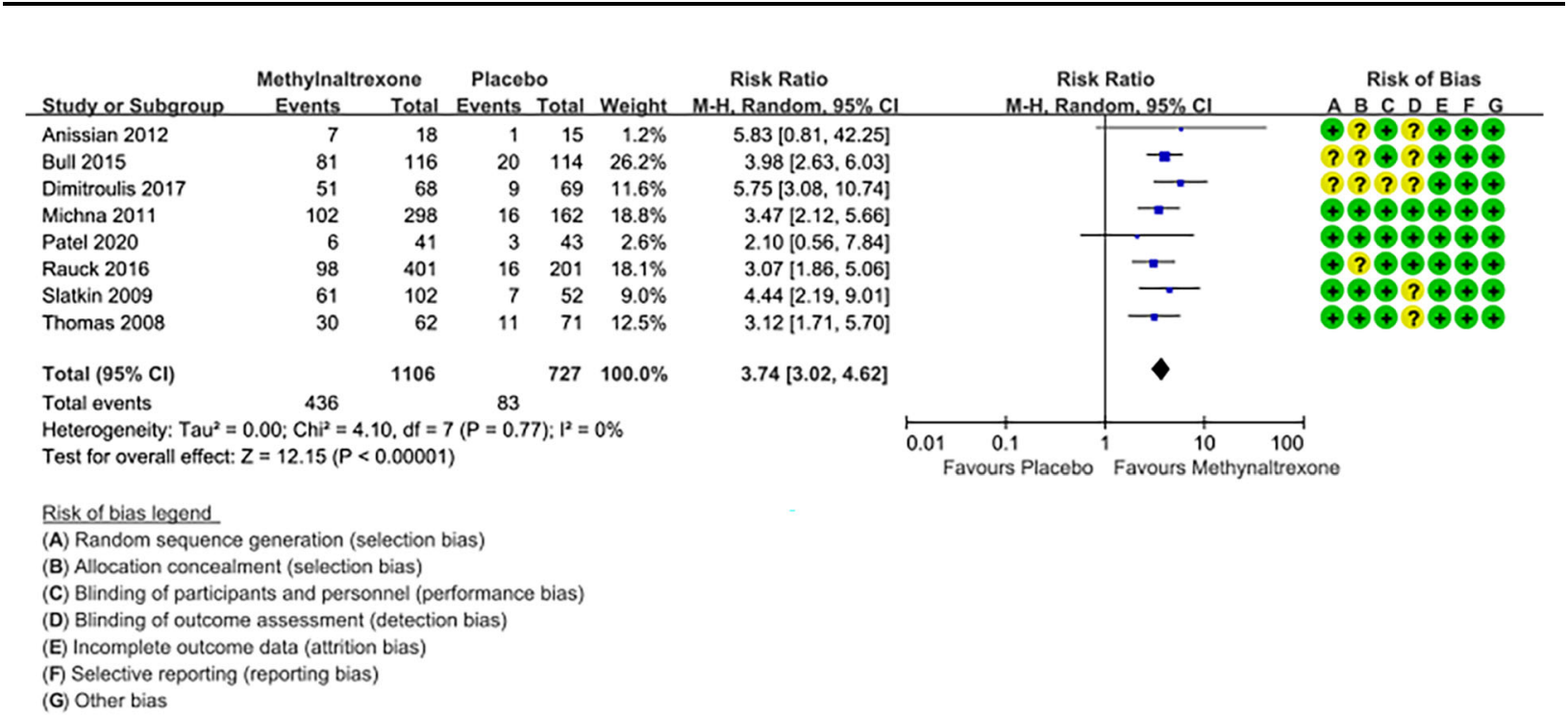

Fig. 3 Forest plot for RFBM within $4 \mathrm{~h}$ after first dose

Secondary Efficacy Outcomes: RFBM Within $24 \mathrm{~h}$ After the First Dose, RFBM $\geq 3$ Times per Week, and Need to Take Rescue Laxatives Compared with placebo, methylnaltrexone significantly increased RFBM within $24 \mathrm{~h}$ after the first dose (two trials; 614 participants; RR 1.98, 95\% CI 1.52-2.58; $I^{2}=9 \%$; Fig. 4) and RFBM $\geq 3$ times per week (three trials; 1396 participants; RR 1.33, 95\% CI 1.17-1.52; $I^{2}=0 \%$; Fig. 4) and decreased need to take rescue laxatives (three trials; 807 participants; RR 0.73, 95\% CI 0.63-0.85; $I^{2}=0 \%$; Fig. 4).

\section{Safety of Methylnaltrexone} for the Treatment of OIC

Primary Safety Outcome: Any Adverse Events Eight trials with 2033 participants reported the primary safety outcome. There was no difference in any adverse events between the methylnaltrexone and placebo groups (RR 1.11, 95\% CI 0.99-1.23; $I^{2}=34 \%$; Fig. 5).

\section{Secondary Safety Outcomes: Abdominal Pain,} Diarrhea, Nausea, Vomiting, and Flatulence

There were no differences in diarrhea (six trials; 1743 participants; RR 1.16, 95\% CI 0.69-1.96; $I^{2}=32 \%$ ), nausea (six trials; 1813 participants; RR 1.15, 95\% CI 0.74-1.79; $I^{2}=23 \%$ ), vomiting (four trials; 977 participants; RR 0.86, 95\% CI
$0.45-1.62 ; I^{2}=25 \%$ ), and flatulence (five trials; 1353 participants; RR 1.41, 95\% CI 0.86-2.32; $I^{2}=0 \%$ ) between the methylnaltrexone and placebo groups (Fig. 6). For the most commonly reported adverse events, the abdominal pain was higher in the methylnaltrexone group than that in placebo group (six trials; 1813 participants; RR 2.30, 95\% CI 1.29-4.08; $I^{2}=62 \%$; Fig. 6).

\section{GRADE Certainty of Evidence}

GRADE evidence profiles for the primary and secondary outcomes are shown in Table 2 . The certainty of evidence is high for RFBM within $4 \mathrm{~h}$ after the first dose, moderate for RFBM within $24 \mathrm{~h}$ after the first dose, RFBM $\geq 3$ times per week, need to take rescue laxatives, any adverse events, abdominal pain, diarrhea, nausea, vomiting, and flatulence.

\section{DISCUSSION}

\section{Main Findings}

Our meta-analysis comprehensively and systematically reviewed the current available literature that compared methylnaltrexone with placebo for treating OIC. We found that 


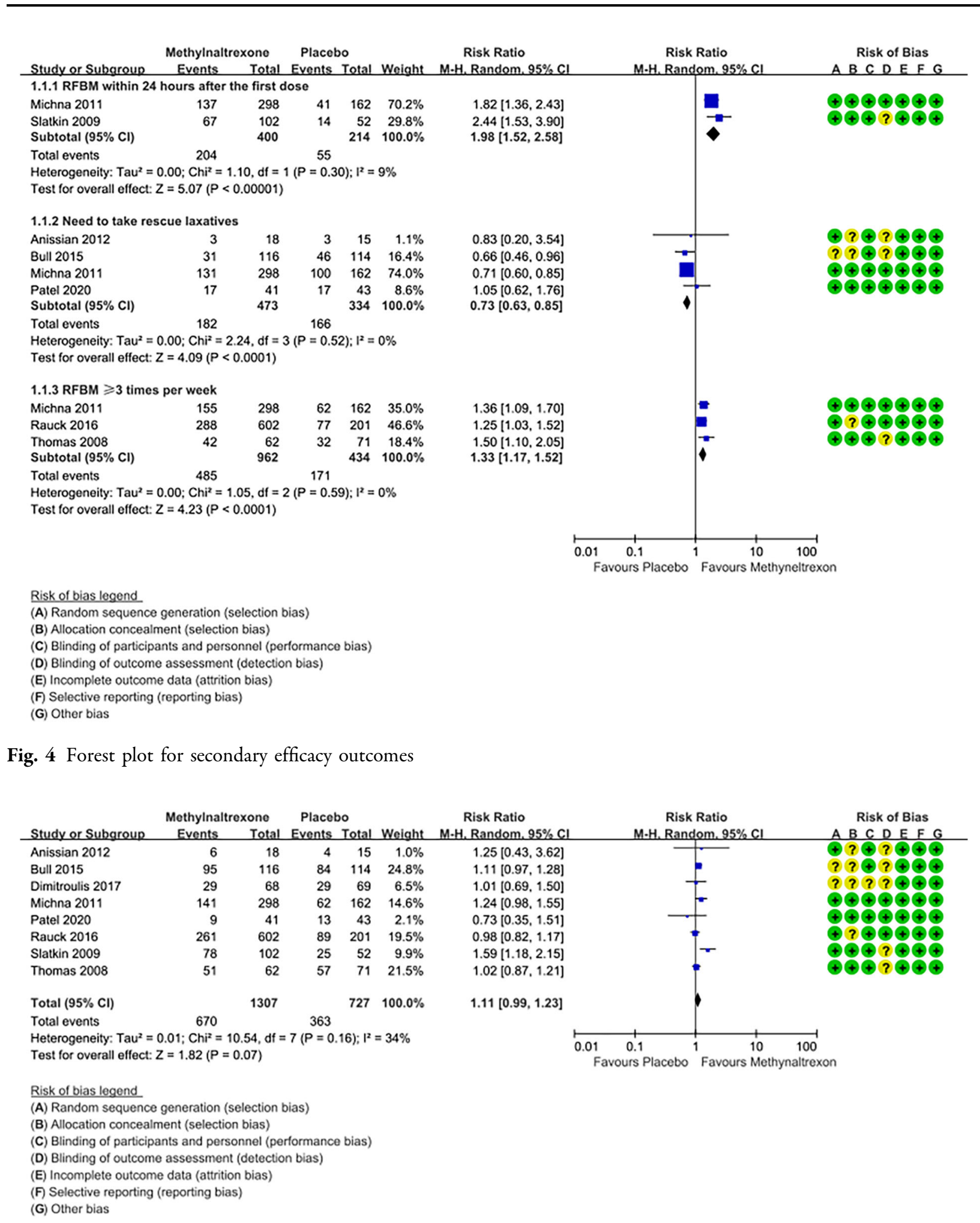

Fig. 5 Forest plot for any adverse events 


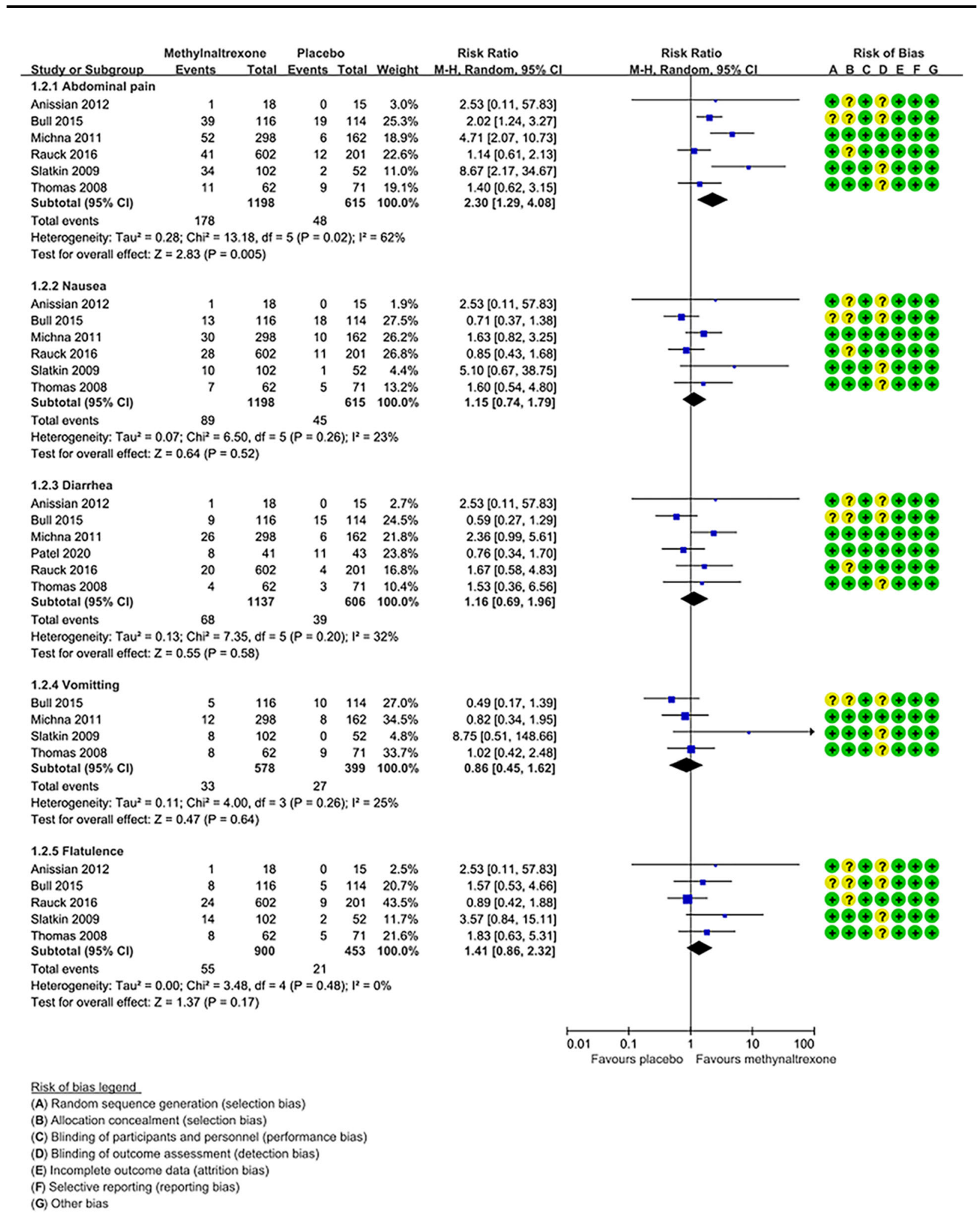

Fig. 6 Forest plot for secondary safety outcomes 


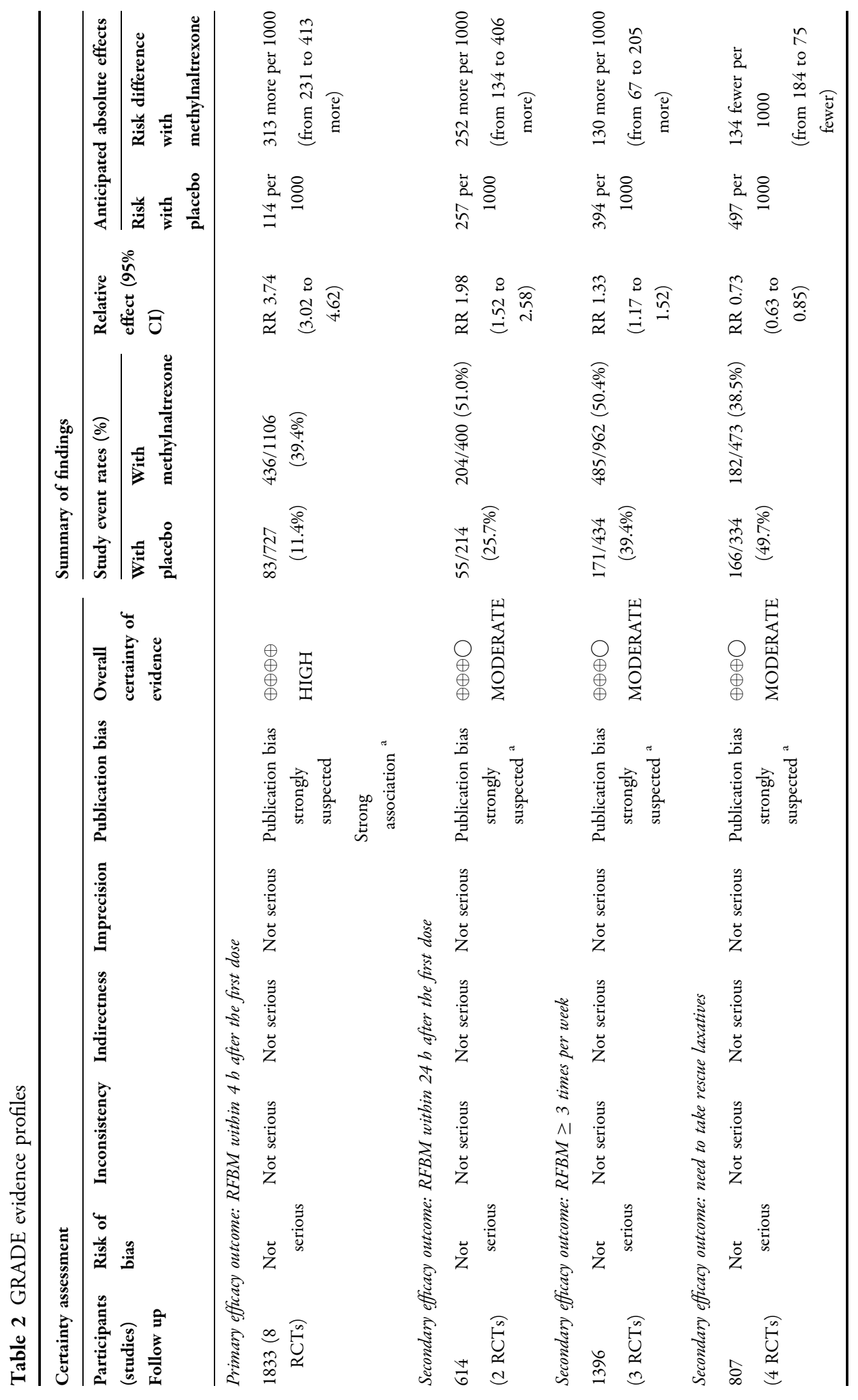




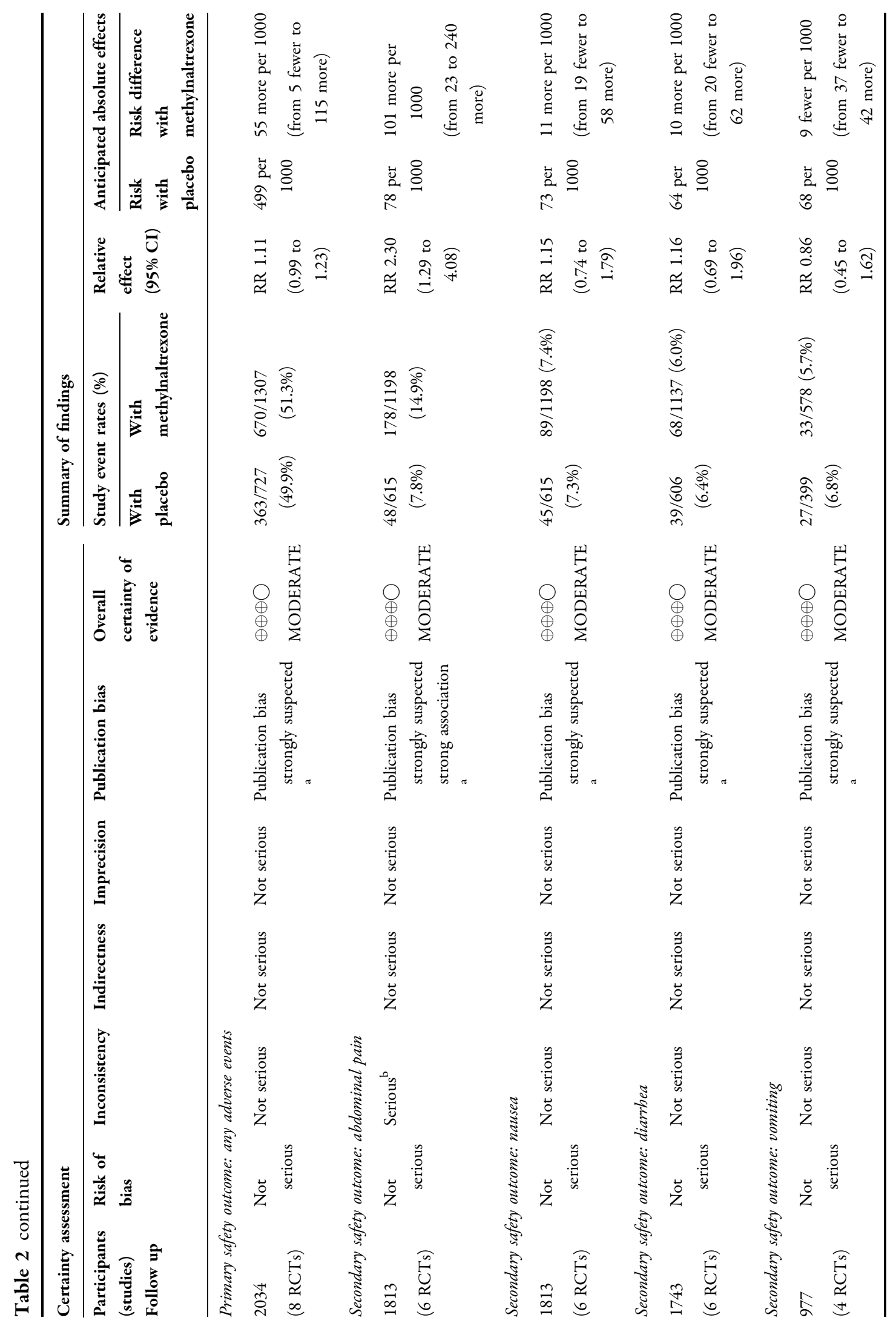




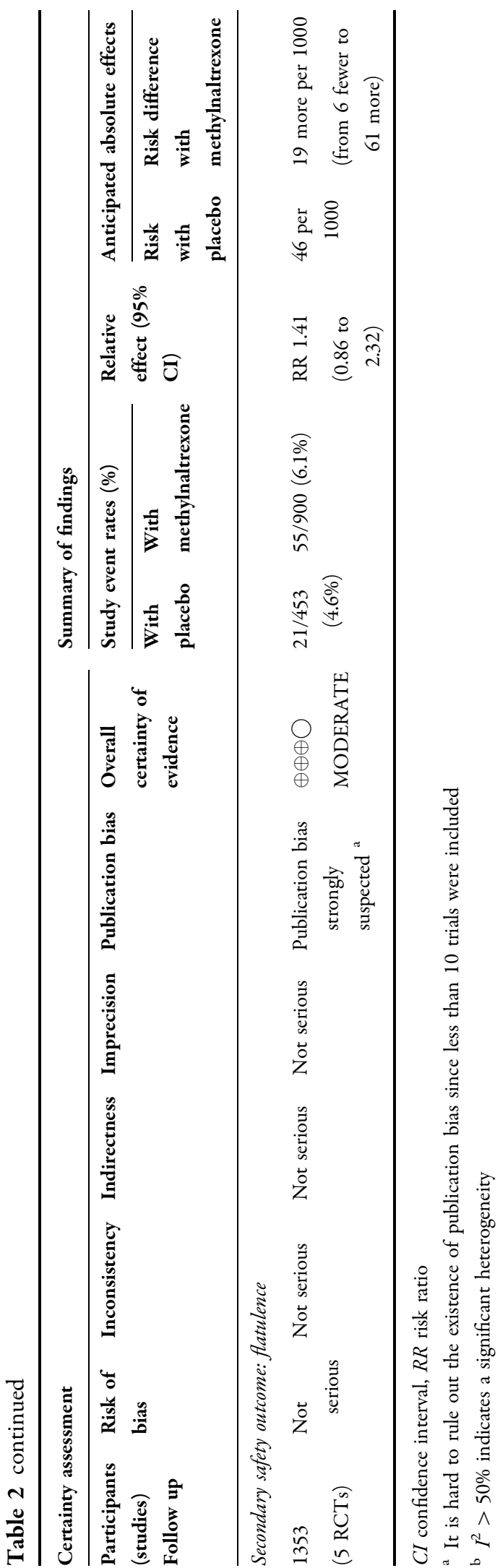

compared with placebo, methylnaltrexone significantly increased RFBM within $4 \mathrm{~h}$ after the first dose, RFBM within $24 \mathrm{~h}$ after the first dose, and $\mathrm{RFBM} \geq 3$ times per week and decreased need to take rescue laxatives; there was no difference in any adverse events (including diarrhea, nausea, vomiting, and flatulence) between the two groups except for abdominal pain.

\section{Comparison with Existing Literature}

Several previous reviews on the similar topic have been published [22-29]. Six of them evaluated the treatment of OIC with different pharmacological therapies, mainly $\mu$-opioid receptor antagonists, including methylnaltrexone [22-27]. These meta-analyses consistently found that methylnaltrexone is effective and safe for the treatment of OIC. Two of them specifically evaluated the effect of methylnaltrexone on the treatment of OIC and found that methylnaltrexone increased RFBM within $4 \mathrm{~h}$ after the first dose $[28,29]$. In line with these two reviews, our meta-analysis also found that methylnaltrexone increased RFBM within $4 \mathrm{~h}$ after the first dose. Besides, we found that methylnaltrexone increased RFBM within $24 \mathrm{~h}$ after the first dose and RFBM $\geq 3$ times per week and decreased need to take rescue laxatives. For safety outcomes, we found that there was no difference in any adverse events (including diarrhea, nausea, vomiting, and flatulence) between the methylnaltrexone and placebo groups. Notably, the occurrence of abdominal pain is higher in the methylnaltrexone group than that in the placebo group. In summary, our meta-analysis further confirmed that methylnaltrexone is an effective and safe drug for the treatment OIC, but some differences also should be noted. First, previous meta-analyses included less than 1500 patients. In comparison, our meta-analysis identified another two recent trials and included more than 2000 patients. With added statistical power of at least 500 cases, our meta-analysis was the latest and the most comprehensive, which further reinforces earlier results of previous meta-analyses. Second, we used an intention-to-treat principle and pooled data with a random-effects model 
accounting for clinical heterogeneity to ensure a more conservative estimate of the efficacy and safety of methylnaltrexone for the treatment of OIC. Third, we evaluated the certainty of evidence using GRADE approach to facilitate clinical decision-making.

\section{Implication for Clinical Practice}

The European expert consensus statement for the management of OIC recommended that peripheral $\mu$-opioid receptor antagonists can be considered as second-line treatment when prophylactics and laxatives are not effective in relieving OIC [30]. The most well-known example is naloxone, commonly used as an intravenous reversal agent in the context of opioid over-dosing. Methylnaltrexone, a quaternary ammonium derivative of naltrexone, has been approved for the treatment of OIC as subcutaneous injection since 2008 . In our metaanalysis, methylnaltrexone was administrated as subcutaneous injection in most trials. The results suggested that methylnaltrexone is effective and safe for the treatment of OIC, but one important thing to note is that methylnaltrexone may increase the risk of abdominal pain. The possible explanations for abdominal pain are as follows: the abdominal pain may be perceived as related to intentional initiative of propulsive peristalsis during the normal process of laxation induced by methylnaltrexone; the abdominal pain may represent localized gastrointestinal withdrawal effects. Although the abdominal pain was mild to moderate and may be dose-dependent, methylnaltrexone still should be used cautiously, especially, in patients with preexisting gastrointestinal disorders. The serious complication of gastrointestinal perforation associated with methylnaltrexone has been reported in some cases [31, 32].

\section{Strengths and Limitations}

The strength of this meta-analysis lies in compliance with the PRISMA statement, registration on PROSPERO with protocol, and applying GRADE approach to assess the certainty of the evidence. Our meta-analysis has some limitations that may affect the interpretation of the results. First, it is hard to rule out the existence of publication bias since only eight trials were included in our meta-analysis. Second, although no statistical heterogeneity was observed for main outcomes, differences in included population and drug regimen may introduce clinical heterogeneity and could affect the results.

\section{CONCLUSIONS}

Methylnaltrexone is an effective and safe drug for treating OIC, but the safety of abdominal pain should be considered.

\section{ACKNOWLEDGEMENTS}

We thank the participants of the study.

Funding. No funding or sponsorship was received for this study or publication of this article. The Rapid Service Fee was funded by the authors.

Authorship. All named authors meet the International Committee of Medical Journal Editors (ICMJE) criteria for authorship for this article, take responsibility for the integrity of the work as a whole, and have given their approval for this version to be published.

Disclosures. Ying-Ying Zhang, Rong Zhou, and Wan-Jie $\mathrm{Gu}$ declare that they have nothing to disclose.

Compliance with Ethics Guidelines. This article is based on previously conducted studies and does not contain any studies with human participants or animals performed by any of the authors.

Data Availability. The datasets generated during and/or analyzed during the current study are available from the corresponding author on reasonable request. 
Open Access. This article is licensed under a Creative Commons Attribution-NonCommercial 4.0 International License, which permits any non-commercial use, sharing, adaptation, distribution and reproduction in any medium or format, as long as you give appropriate credit to the original author(s) and the source, provide a link to the Creative Commons licence, and indicate if changes were made. The images or other third party material in this article are included in the article's Creative Commons licence, unless indicated otherwise in a credit line to the material. If material is not included in the article's Creative Commons licence and your intended use is not permitted by statutory regulation or exceeds the permitted use, you will need to obtain permission directly from the copyright holder. To view a copy of this licence, visit http://creativecommons.org/licenses/by$\mathrm{nc} / 4.0 /$.

\section{REFERENCES}

1. Tokoro A, Imai H, Fumita $S$, et al. Incidence of opioid-induced constipation in Japanese patients with cancer pain: a prospective observational cohort study. Cancer Med. 2019;8:4883-91.

2. Kalso E, Edwards JE, Moore RA, McQuay HJ. Opioids in chronic non-cancer pain: systematic review of efficacy and safety. Pain. 2004;112:372-80.

3. Fine PG, Chen YW, Wittbrodt E, Datto C. Impact of opioid-induced constipation on healthcare resource utilization and costs for cancer pain patients receiving continuous opioid therapy. Support Care Cancer. 2019;27:687-96.

4. Wittbrodt ET, Gan TJ, Datto C, McLeskey C, Sinha $\mathrm{M}$. Resource use and costs associated with opioidinduced constipation following total hip or total knee replacement surgery. J Pain Res. 2018;11: 1017-25.

5. Argoff CE, Brennan MJ, Camilleri M, et al. Consensus recommendations on initiating prescription therapies for opioid-induced constipation. Pain Med. 2015;16:2324-37.

6. Candy B, Jones L, Larkin PJ, Vickerstaff V, Tookman A, Stone P. Laxatives for the management of constipation in people receiving palliative care. Cochrane Database Syst Rev. 2015;2015:CD003448.
7. Nelson AD, Camilleri M. Opioid-induced constipation: advances and clinical guidance. Ther Adv Chronic Dis. 2016;7:121-34.

8. Higgins JPT, Altman DG, editors. Cochrane handbook for systematic reviews of interventions. Version 5.0.1. The Cochrane Collaboration; 2008.

9. Moher D, Liberati A, Tetzlaff J, Altman DG; PRISMA Group. Preferred reporting items for systematic reviews and meta-analyses: the PRISMA statement. BMJ. 2009;339:b2535.

10. Higgins JP, Altman DG, Gøtzsche PC, et al, Cochrane Bias Methods Group, Cochrane Statistical Methods Group. The Cochrane Collaboration's tool for assessing risk of bias in randomised trials. BMJ. 2011;343:d5928.

11. Guyatt GH, Oxman AD, Vist GE, et al, GRADE Working Group. GRADE: an emerging consensus on rating quality of evidence and strength of recommendations. BMJ. 2008;336:924-6.

12. Higgins JP, Thompson SG, Deeks JJ, Altman DG. Measuring inconsistency in meta-analyses. BMJ. 2003;327:557-60.

13. Higgins JP, Thompson SG. Quantifying heterogeneity in a meta-analysis. Stat Med. 2002;21: 1539-58.

14. Thomas J, Karver S, Cooney GA, et al. Methylnaltrexone for opioid-induced constipation in advanced illness. N Engl J Med. 2008;358:2332-43.

15. Slatkin N, Thomas J, Lipman AG, et al. Methylnaltrexone for treatment of opioid-induced constipation in advanced illness patients. J Support Oncol. 2009;7:39-46.

16. Michna E, Blonsky ER, Schulman S, et al. Subcutaneous methylnaltrexone for treatment of opioidinduced constipation in patients with chronic, nonmalignant pain: a randomized controlled study. J Pain. 2011;12:554-62.

17. Anissian L, Schwartz HW, Vincent K, et al. Subcutaneous methylnaltrexone for treatment of acute opioid-induced constipation: phase 2 study in rehabilitation after orthopedic surgery. J Hosp Med. 2012;7:67-72.

18. Bull J, Wellman CV, Israel RJ, Barrett AC, Paterson C, Forbes WP. Fixed-dose subcutaneous methylnaltrexone in patients with advanced illness and opioid-induced constipation: results of a randomized, placebo-controlled study and open-label extension. J Palliat Med. 2015;18:593-600.

19. Rauck R, Slatkin NE, Stambler N, Harper JR, Israel RJ. Randomized, double-blind trial of oral 
methylnaltrexone for the treatment of opioid-induced constipation in patients with chronic noncancer pain. Pain Pract. 2017;17:820-8.

20. Dimitroulis I, Peristeris P, Toumbis M. Effectiveness of methylnaltrexone bromide in opioid-induced constipation in advanced NSCLC patients. J Thorac Oncol. 2017;12:S2232.

21. Patel PB, Brett SJ, O'Callaghan D, et al. Methylnaltrexone for the treatment of opioid-induced constipation and gastrointestinal stasis in intensive care patients. Results from the MOTION trial. Intensive Care Med. 2020;46:747-55.

22. McNicol E, Boyce DB, Schumann R, Carr D. Efficacy and safety of mu-opioid antagonists in the treatment of opioid-induced bowel dysfunction: systematic review and meta-analysis of randomized controlled trials. Pain Med. 2008;9:634-59.

23. Ford AC, Brenner DM, Schoenfeld PS. Efficacy of pharmacological therapies for the treatment of opioid-induced constipation: systematic review and meta-analysis. Am J Gastroenterol. 2013;108: 1566-74.

24. Candy B, Jones L, Vickerstaff V, Larkin PJ, Stone P. Mu-opioid antagonists for opioid-induced bowel dysfunction in people with cancer and people receiving palliative care. Cochrane Database Syst Rev. 2018;6:CD006332.

25. Nee J, Zakari M, Sugarman MA, et al. Efficacy of treatments for opioid-induced constipation: systematic review and meta-analysis. Clin Gastroenterol Hepatol. 2018;16:1569-84.
26. Vijayvargiya $\mathrm{P}$, Camilleri $\mathrm{M}$, Vijayvargiya $\mathrm{P}$, Erwin $\mathrm{P}$, Murad MH. Systematic review with meta-analysis: efficacy and safety of treatments for opioid-induced constipation. Aliment Pharmacol Ther. 2020;52:37-53.

27. Ouyang R, Li Z, Huang S, Liu J, Huang J. Efficacy and safety of peripherally acting mu-opioid receptor antagonists for the treatment of opioid-induced constipation: a Bayesian network meta-analysis. Pain Med. 2020. https://doi.org/10.1093/pm/ pnaa152.

28. Mehta N, O'Connell K, Giambrone GP, Baqai A, Diwan S. Efficacy of methylnaltrexone for the treatment of opioid-induced constipation: a metaanalysis and systematic review. Postgrad Med. 2016;128:282-9.

29. Siemens W, Becker G. Methylnaltrexone for opioidinduced constipation: review and meta-analyses for objective plus subjective efficacy and safety outcomes. Ther Clin Risk Manag. 2016;12:401-12.

30. Farmer AD, Drewes AM, Chiarioni G, et al. Pathophysiology and management of opioid-induced constipation: European expert consensus statement. United Eur Gastroenterol J. 2019;7:7-20.

31. Blackney KA, Kamdar NV, Liu CA, Edwards DA. Methylnaltrexone-associated bowel perforation in postoperative opioid-induced constipation and Ogilvie syndrome: a case report. A A Pract. 2019;12: 44-6.

32. Mackey AC, Green L, Greene P, Avigan M. Methylnaltrexone and gastrointestinal perforation. J Pain Symptom Manag. 2010;40:e1-3. 